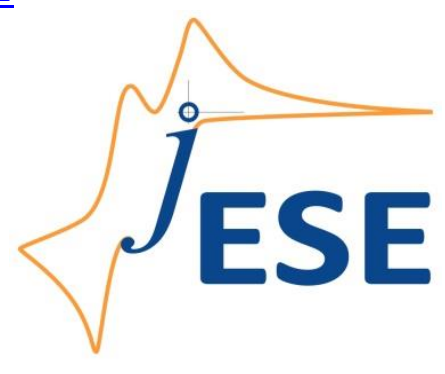

Open Access: ISSN 1847-9286

www.jESE-online.org

Original scientific paper

\title{
Electrochemical reduction of artemisinin: Chromatographic identification of bulk electrolysis products
}

\author{
Faraja Obwayo, Zahilis Mazzochette, Amos Mugweru ${ }^{\bowtie}$ \\ Department of Chemistry and Biochemistry, Rowan University, Glassboro, NJ 08028, USA \\ ${ }^{\bowtie}$ Corresponding authors: mugweru@rowan.edu; Tel.: +1-856-2565454; Fax: +1-856-256-4478
}

Received: January 4, 2019; Revised: March 15, 2019; Accepted: March 18, 2019

\begin{abstract}
Artemisinin is a naturally occurring sesquiterpene lactone with an endo-peroxide bond. This drug is used for treatment of many diseases including malaria. The reduction of this molecule on an electrode surface was carried out by cyclic voltammetry as well as amperometry. Cyclic voltammetry of artemisinin generated one prominent peak wave at $-1.0 \mathrm{~V}$ and another, smaller one at $-0.3 \mathrm{~V} v \mathrm{sg} / \mathrm{AgCl}$ reference electrode. The bulk electrolysis of artemisinin on a carbon electrode generated two other irreversible peak waves at around -0.7 and $-0.1 \mathrm{~V}$. The concentration of the products was dependent on the time of electrolysis. LC-MS was used to determine the bulk electrolysis products of artemisinin. Initially dihydroartemisinin was generated as the main reduction product. Other reduction products were formed after further reduction of dyhidroartemisinin.
\end{abstract}

\section{Keywords}

Cyclic voltammetry; dyhidroartemisinin; electrochemical reduction; bulk electrolysis; LC-MS

\section{Introduction}

Artemisinin is a commonly used drug for the control of malaria [1]. It occurs naturally and can be extracted from artemisia flowers. The artemesia plants are grown in several countries including Africa and Asia. Artemisinin (ART) has been in use in traditional Chinese medicine for thousands of years for the treatment of fever and other related diseases [2,3]. The mature artemisia flowers are gold tainted and together with the seeds have strong anti-malaria effect. In some parts of Africa and Asia, the flowers and seeds are used as herbal tea after drying. Even when a small number of flowers are left in a glass of water for some time at room temperature, a delayed extraction of ART takes place, capable of malaria prevention. Thus, these flowers provide a strong treatment for malaria attack in remote areas, where anti malaria drugs are not available. Malaria is a devastating disease that kills many people every year. 
Some of ART derivatives are given in Figure 1. They all have one thing in common, the endoperoxide bridge. The mechanism of action of ART is not well understood but a possible mechanism is postulated to involve the heme-mediated decomposition of the endo-peroxide bond to produce free radicals which kill the parasites accumulated in the erythrocytes [4]. Recent studies suggest that this medicine can induce toxicity to male germ cells which may have implications in male reproductive toxicity [5].

ART is of current major interest among scientists, trying to understand its nature and properties. Different analytical techniques have been used to study this drug. Understanding the nature and mode of action of this drug is essential in light of the recent reports from parts of Asia suggesting that malaria is becoming more resistant to ART [6-8]. On the other hand, there are reports suggesting that ART possess anticancer properties $[9,10]$.

The methods developed for the determination of ART include high performance or ultraperformance liquid chromatography [11], molecularly imprinted electrodes [12], polymerized molecularly imprinted membranes [13] among others. ART contains an electrochemically active endo-peroxide group that can be selectively measured by using electroanalytical methods on various electrode surfaces [14]. The goal of the current work was to obtain a detailed electrochemical and chromatographic characterization of ART bulk electro-reduction products. Previously, some of the electro-oxidation products on the electrode surface matched the enzyme generated metabolites of some other drug molecules [15].

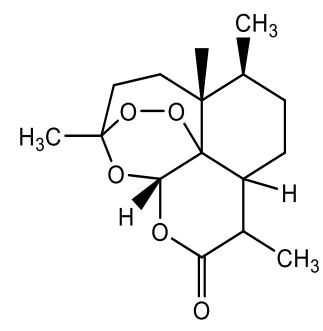

Artemisinin
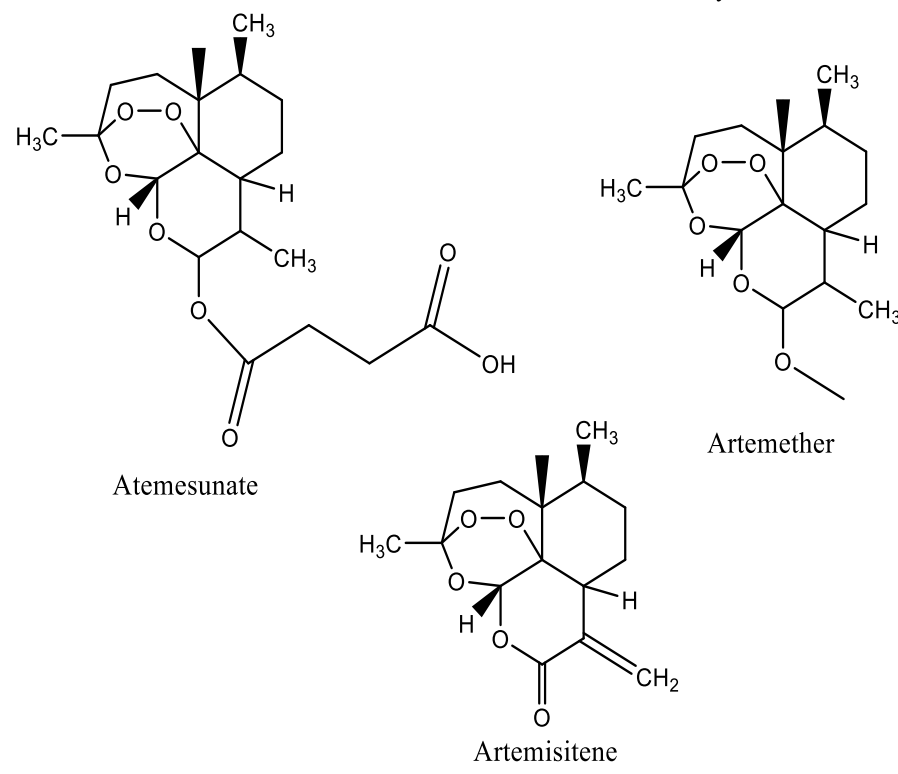

Figure 1. Chemical structures of artemisinin and its derivatives

Feng Zhang et al. used cyclic voltammetry to show that ART was irreversibly reduced at approximately $-1.0 \mathrm{~V}$ vs. $\mathrm{Ag} / \mathrm{AgCl}$ reference electrode [16]. Presence of hemin was found to lower 
the reduction potential significantly to $-0.435 \mathrm{~V} v s$. $\mathrm{Ag} / \mathrm{AgCl}$. However, in this paper, instability of ART in presence of hemin was noted. Using cyclic and differential pulse voltammetry and in phosphate buffer of $\mathrm{pH} \mathrm{7,} \mathrm{carbon} \mathrm{hemin} \mathrm{modified} \mathrm{electrode} \mathrm{indicated} \mathrm{ART} \mathrm{reduction} \mathrm{potential} \mathrm{of}$ about -380 mV vs. Ag/AgCl. However, film modified multi-wall carbon nanotube (MWNT) increased reduction peak current of ART remarkably and the peak potential shifted positively by about -240 $\mathrm{mV}$ [17]. ART has also been studied using a gold amalgam and gold electrode [18] and also with [FeT(o-glu)TTCl]/Au nanoparticles modified electrode [19].

Other studies suggest that the electrochemical reduction of ART is two electron process at about $-0.9 \mathrm{~V}$ vs. $\mathrm{Ag} / \mathrm{AgCl}$ [20-22]. This electrochemical reaction is an irreversible process likely involving heterolytic cleavage of the $\mathrm{O}-\mathrm{O}$ bond $[20,23]$. This reaction can be carried out using a controlled potential electrosynthesis (bulk electrolysis). Controlled-potential electrolysis involves application of a selected potential for a prescribed period of time (as the current decays exponentially). Bulk electrolysis offers unique selectivity. There is increased interest in the application of electrochemical techniques for specific synthesis of organic molecules [24-26] and environmental remediation [27]. It is of interest to measure the actual products as well as their rate of formation. Such information is important in correlating the mechanism of drug action in vivo. ART and some of its derivatives are known to affect the function of cytochrome P450's [28].

In the present work, the electro-synthesis of ART and some of its derivatives has been studied. Electrochemistry induced organic reaction represents a versatile method for generating new molecules and possibly providing plausible mechanisms of enzymatic mediated metabolism. There are no previous reports on the electro-synthesis involving ART or its derivatives. There are several known derivatives of ART including dihydroartemisinin, artemether, arteether and artesunate. Dihydroartemesinin is also one of the clinically useful metabolite of ART. Detailed characterization of the electrochemical reduction products provides fundamental information about the direct electron transfer processes involved in ART electro-oxidation.

\section{Experimental}

\section{Materials and reagents}

ART was purchased from Tokyo Chemical Industry CO., LTD. methanol, and tetra butyl ammonium bromide ( $\left.\mathrm{Bu}_{4} \mathrm{NBr}-\mathrm{TBAB}\right)$ were purchased from WWR (West Chester, $\mathrm{PA}$ ). Solutions of ART containing $0.05 \mathrm{M}$ tetrabutyl ammonium bromide (TBAB) in acetonitrile were prepared prior to each experiment. The TBAB was used as a supporting electrolyte. All other chemicals were HPLC grade.

\section{Electrochemical apparatus}

Amperometric and cyclic voltammetry (CV) techniques were carried out with a computer controlled electrochemical workstation ( $\mathrm{CHI} 660 \mathrm{c}$, USA) with $98 \%$ ohmic drop (IR) compensation. A three-electrode electrochemical cell was used for all electrochemical experiments. Glassy carbon (area, $0.017 \mathrm{~cm}^{2}$ ) was used as the working electrode, and a platinum wire as the counter electrode. The working and the counter electrodes were obtained from Bioanalytical Systems Inc. (West Lafayette, IN). An $\mathrm{Ag} / \mathrm{AgCl}$, equipped with a glass tip, separated from the sample solution compartment by a salt-bridge containing $\mathrm{KCl}$ and terminating in a medium porosity glass frit, was used as the reference electrode. For bulk electrolysis experiments, vitreous carbon electrode was used as a working electrode. All electrochemical measurements were carried out at ambient conditions. 


\section{Bulk electrolysis procedure}

The reduction reaction of ART was carried out in ammonium bromide-methanol solution under stirring conditions. A bulk electrolysis experiment required a large surface area working electrodes in order to increase the rate of electrolysis. In this work, a reticulated vitreous carbon electrode was used as the working electrode. The electrolyte contained $0.05 \mathrm{M}$ TBAB in methanol. The electrode potential during electrolysis was fixed at $-1.5 \mathrm{~V}$ vs. $\mathrm{Ag} / \mathrm{AgCl} .0 .5 \mathrm{~mL}$ aliquots of reaction mixture were withdrawn every hour, filtered and analyzed using liquid chromatographic techniques. The solvents used were compatible with mobile phase for separation and hence, no extraction was needed.

\section{Chromatographic procedure}

Separation of bulk electrolysis reduction products were carried out using ZORBAX C-18 300 SB column $(4.6 \times 150 \mathrm{~mm})$ and with $3.5 \mu \mathrm{m}$ particle size, Agilent Technologies. The mobile phase consisted of a combination of acetonitrile, methanol and de-ionized water (50:25:25) respectively with $0.1 \%$ formic acid. The HPLC-UV analysis was carried out using Agilent 1100 series HPLC unit equipped with an UV-Visible detector with the wavelength set at $254 \mathrm{~nm}$. The chromatographic run were performed under isocratic conditions at the flow rate of $100 \mu \mathrm{L} / \mathrm{min}$. Every end of the run was followed by a post run step that included flushing the column with mobile phase repeatedly.

\section{Mass spectrometer procedure}

The mass spectrometry detection for the LC/MS was an Ion Trap 1100 series. The mass spectrometer was set to ESI in positive ion mode. The settings of the mass spectrometer were as follows: Scan mode (standard), range $(50-2200) \mathrm{m} / \mathrm{z}$, speed $(13,000 \mathrm{~m} / \mathrm{z} / \mathrm{min})$, threshold (1000), nebulizer gas $(15.0 \mathrm{psi})$, and dry gas $(5.0 \mathrm{~L} / \mathrm{min})$, dry temp $\left(325^{\circ} \mathrm{C}\right)$. The target mass was set to 282 while the compound stability and trap drive level were set to $100 \%$. The ramp range was from 4500.0 to $1500.0 \mathrm{~V}$. The $\mathrm{ms} / \mathrm{ms}$ fragmentation amp was set to $1.00 \mathrm{~V}$.

\section{Results and discussion}

\section{Electrochemical reduction of ART}

Previously, we reported kinetic parameters for direct electrochemical reduction of ART on gold electrodes [29]. We also reported electrochemical oxidation on carbon nanofibers modified with hemoglobin [30]. The rates of electron transfer on gold electrodes and the diffusion coefficient during the electroreduction were determined. Here, we have studied the bulk electrolysis of ART and attempted to assign molecular structures of possible products.

Figure 2 shows cyclic voltammograms of ART analysed using a glassy carbon electrode before and after bulk electrolysis procedure. Electrolyzed ART generated four irreversible redox peak waves. Before electrolysis (time zero), a prominent peak wave was observed at around -1.0 while another smaller peak appeared at $-0.3 \mathrm{~V}$. After 60 minutes of electrolysis, a second peak wave developed at around $-0.7 \mathrm{~V}$. Another irreversible peak was also abserved at $-0.10 \mathrm{~V}$. The peak currents of these new peak waves increased with increase of time of electrolysis. The peaks shifted in the positve direction by about $0.3 \mathrm{~V}$ in about 4 hours of electrolysis. Scan rate dependence studies were carried out ( 0 to $1000 \mathrm{mV} / \mathrm{s}$ ) using glassy carbon electrode and fresh ART solution. Figure 3 shows the cyclic voltammograms obtained. Scan rate dependence study showed two pronounced oxidation peaks at $-0.7 \mathrm{~V}$ and another at $0.2 \mathrm{~V}$. Increased scan rates resulted in increaased peak currents for all the four peak waves. 


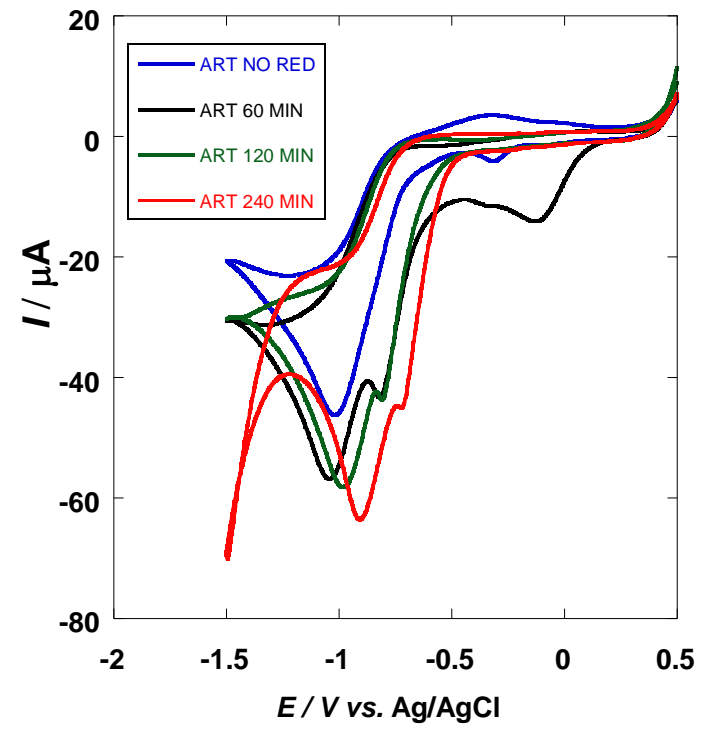

Figure 2: Cyclic voltammograms of $0.03 \mathrm{MART}$ before and after 1, 2 and 4 hours of electrolysis.

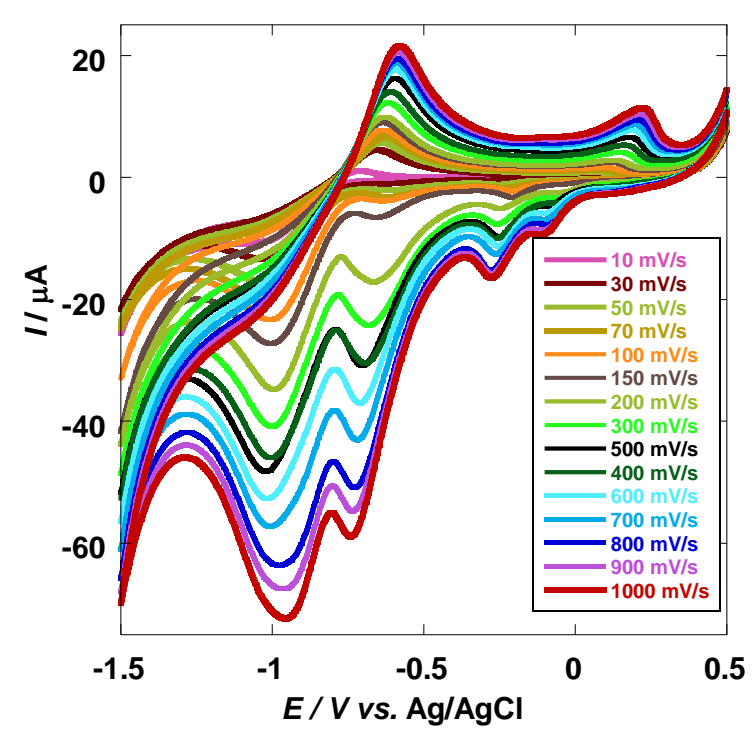

Figure 3. Cyclic voltammograms of $0.03 \mathrm{M}$ ART taken at different scan rates $(10-1000 \mathrm{mV} / \mathrm{s}$

Reduction peak waves occurring at about $0.1,-0.3,-0.7$ and $-1.0 \mathrm{~V} v \mathrm{vs} . \mathrm{Ag} / \mathrm{AgCl}$ show clear increase of current as a function of scan rate. These four peaks were not previously observed while carrying out electrochemistry in acetonitrile solution. Figure 4 shows current peak height plotted as a function of the scan rate. At scan rate below $150 \mathrm{mV} / \mathrm{s}$, peak current and scan rate show a linear relationship. However, at high scan rates, (above $200 \mathrm{mV} / \mathrm{s}$ ), the peak especially one occurring at about $-1.0 \mathrm{~V}$ plateaued and was no longer linear with scan rate (Figure 4). Plots of peak current as a function of square-root of the scan rates show linear relationships. Under diffusion control, a plot of $I_{\mathrm{p}}$ versus $v^{1 / 2}$ is usually linear. This implied that ART reduction on the electrode is under diffusion control.
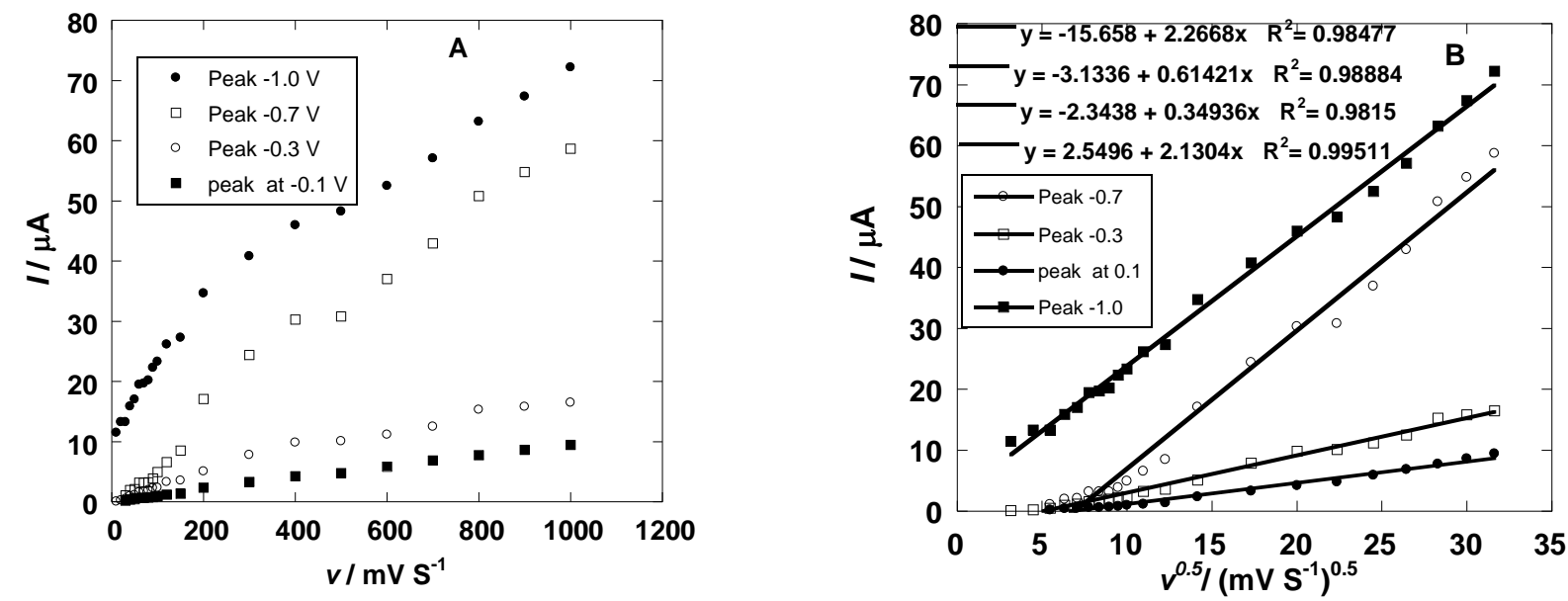

Figure 4. $0.03 \mathrm{M}$ artemisinin in methanol containing 0.05M TBAB at glassy carbon electrode $A$ - Peak currents as a function of scan rate (10-1000 $\left.\mathrm{mVs}^{-1}\right)$;

$B$ - Peak current as a function of square root of scan rate.

From previous studies of electrochemical catalysis of ART on electrodes functionalized with hemoglobin [30], it was possible to quantitate the ART oxidation or reduction products and at the same time estimate the kinetics of the reaction. Both the electrode oxidation and reduction reactions of ART are irreversible. From cyclic voltammograms in Figures 2 and 3, we observe four reduction wave forms and two oxidation wave forms, that indicates that six different species in the solution could have been formed. In order to investigate these species being formed, we fixed the 
potential of the reaction at $-1.5 \mathrm{~V} v \mathrm{vs} . \mathrm{Ag} / \mathrm{AgCl}$ and withdrew $100 \mu \mathrm{L}$ aliquots of reaction mixture every hour for chromatographic analysis. Although some of the products could be transient, it's possible to determine some compounds that can provide a road map to the possible mechanisms involved.

\section{Chromatography}

Bulk electrolysis of $0.03 \mathrm{M}$ ART solution using a vitreous carbon electrode involved maintaining the electrode at $-1.5 \mathrm{~V} v \mathrm{~s}$. $\mathrm{Ag} / \mathrm{AgCl}$ for up to 4 hours. The products of electro-oxidation were obtained in a stirred solution and filtered before analysis by chromatography. The solution was stirred during $0.5 \mathrm{~mL}$ aliquots of the solution were drawn using a syringe each hour during the electrolysis. The aliquots were filtered using a $0.22 \mathrm{~nm}$ filter and injected onto a chromatographic instrument. The HPLC was equipped with UV detector. Chromatograms of different solutions are given on Figure 5. At time zero (before any electrolysis), only one chromatographic peak was obtained at about 25.5 minutes. This is the chromatogram of unaltered ART (red chromatogram). On subjecting the ART solution to bulk electrolysis, four more peaks appeared at $22.5 \mathrm{~min} 21.5 \mathrm{~min}$, 18.0 and 27.5 minutes (Figure 5). However, the highest peak was at 22.5 minutes. The first reduction peak wave was later confirmed using LC-MS to be due to the formation of dihydroartemisinin, while the second and third peaks were other reduction products of artemisinin as described below.

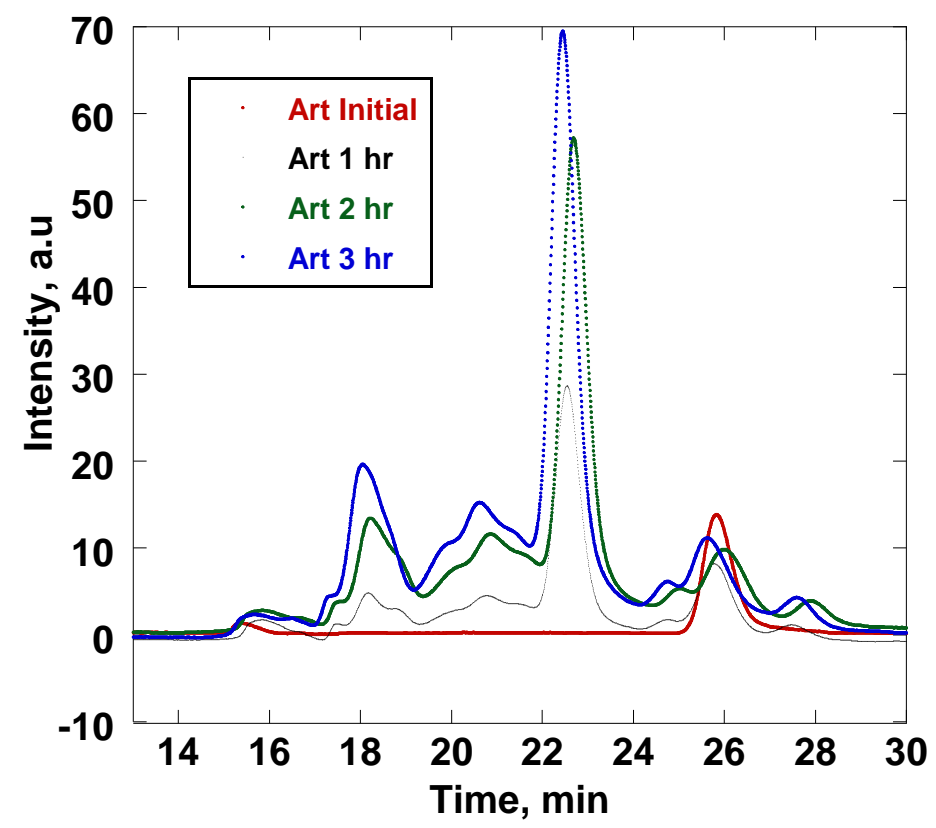

Figure 5. Chromatograms of bulk electrolysis of ART at $-1.5 \mathrm{~V} v \mathrm{vs} . \mathrm{Ag} / \mathrm{AgCl}$ at different durations.

Other chromatographic peaks appeared after one hour of electrolysis. The new significant peaks appeared at about 22.5, 21.5 and 18 minutes. These new peaks grew with time of the electrolysis. In a bid to establish the identity of the new electroreduction products, the electrolyzed samples were injected into LC-Ms instrument using same column and mobile phase composition.

Dyhidroartemisinin is one of the products of artemether metabolism by cytochrome P450 isoenzyme 3A4 [31]. There might be a relationship between the electrode electrochemical transformation or ART and the pharmacokinetic of the ART family. Results of electrochemical reduction of these drugs can partly unravel potential metabolites. The probable initial electrochemical reduction product of ART is dyhidroartemisinin shown in Figure 6 . The aldehyde group at position 11 is converted to alcohol during the electrochemical reduction. We believe that 
it is the first step in electrochemical reduction. The second step is dihydroartemesinin undergoing an electrochemical reaction to generate an intermediate species involving heterolytic cleavage of the $\mathrm{O}-\mathrm{O}$ bond. The intermediate products can later undergo further chemical re-organization to generate a final multiple products. Three chromatographic peak waves are observed. The electrocatalytic behavior observed for artemisinin reduction points to ECE catalytic mechanism.

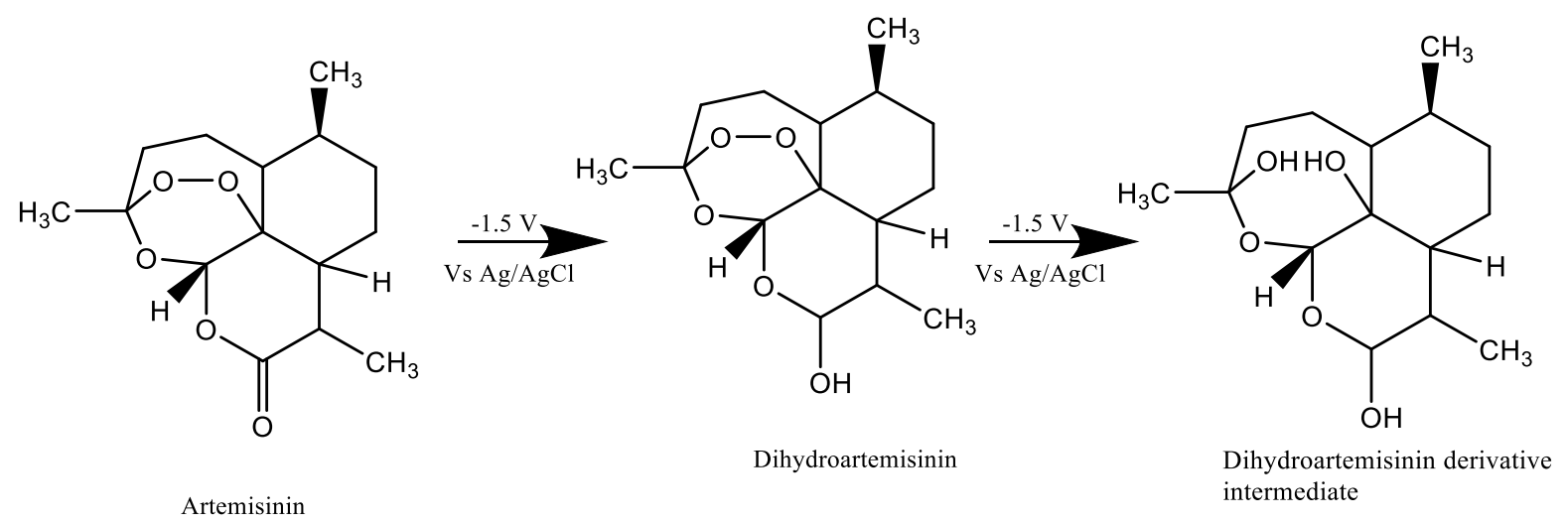

Figure 6. Possible ART electrochemical reduction reaction mechanism during bulk electrolysis at -1.5 V.

Figure 7 shows a mass spectrum of the initial ART peak obtained at 25.5 minutes. The initial drug has well known fragments with $\mathrm{m} / \mathrm{z}$ of the molecular ion [M] ${ }^{+}$at 282 , while other fragments include molecular ion with a sodium ion. The presence of other ions above the protonated molecular ion at $\mathrm{m} / \mathrm{z} 299, \mathrm{~m} / \mathrm{z} 300$ and $\mathrm{m} / \mathrm{z} 305$ on the mass spectrum point to adducted ions of clustered methanol and sodium to the ART, i.e $\mathrm{m} / \mathrm{z} 305$ for $[\mathrm{M}+\mathrm{Na}]^{+}, \mathrm{m} / \mathrm{z} 283$ for $[\mathrm{M}+\mathrm{H}]^{+}$, and $\mathrm{m} / \mathrm{z} 299$ for $\left[\mathrm{M}+\mathrm{NH}_{4}{ }^{+}\right]$.

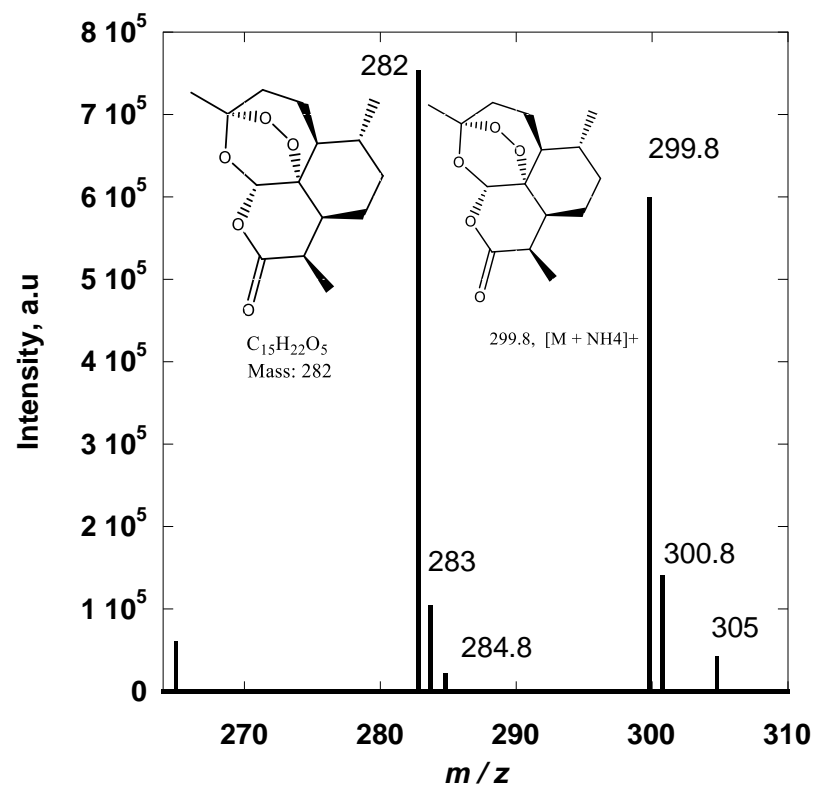

Figure 7. Mass spectrum of ART before electrolysis (peak at around 25.5 minutes)

Figure 8 shows the mass spectrum for the chromatographic peak at 22.5 minutes after one hour of electro-reduction. This new chromatographic peak is one of the new major products of ART electro-reduction. The growth of this peak was steady with time of electrolysis as observed from the increasing peak height with increasing time of bulk electro-reduction. The fragment with $\mathrm{m} / \mathrm{z} 284$ is most likely the molecular ion of dihydroartemisinin. The fragments of this molecule were found to be $283,267,300$ and 265 and 258 . The fragments obtained are given below. 

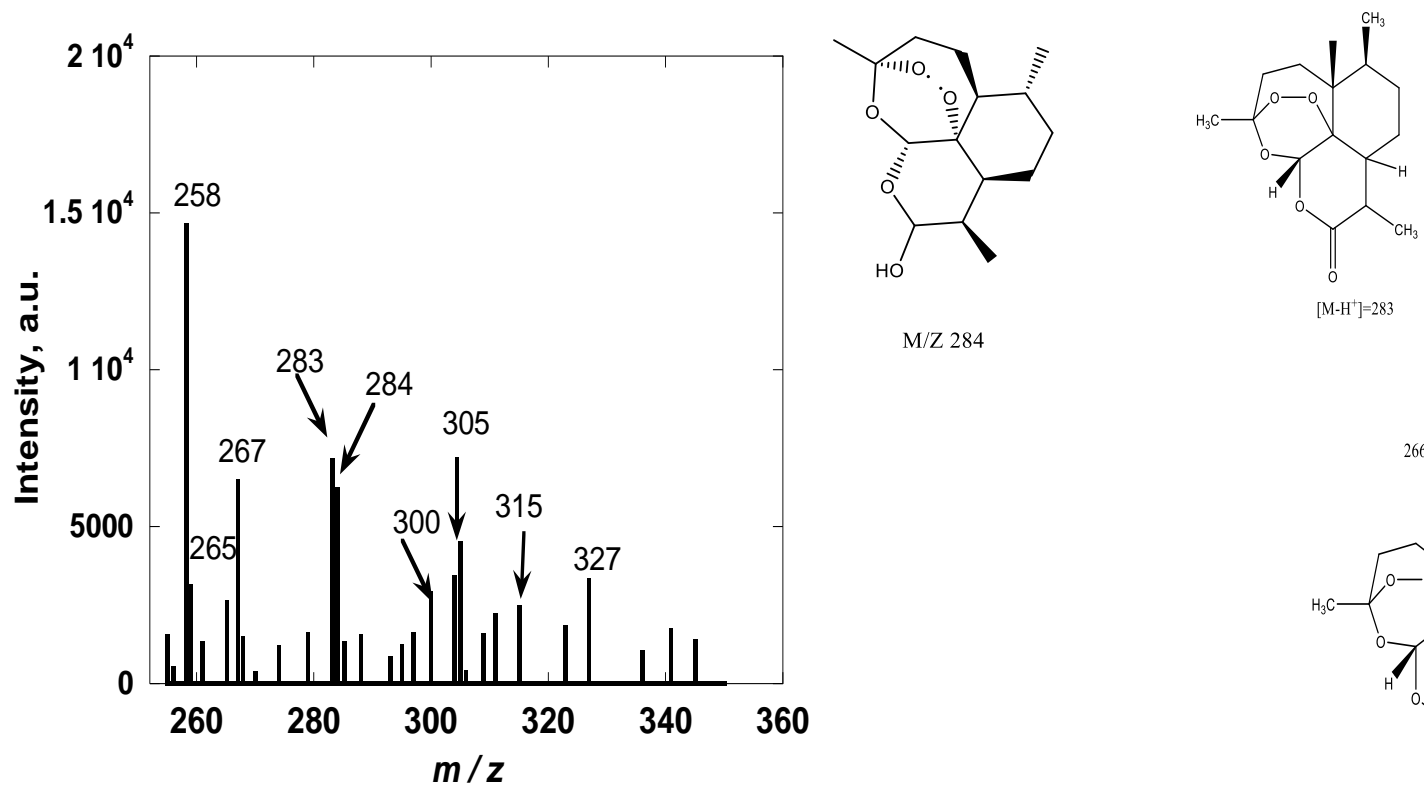

Figure 8. Mass spectrum of ART after one hour of electrolysis (peak at $22.5 \mathrm{~min}$ )

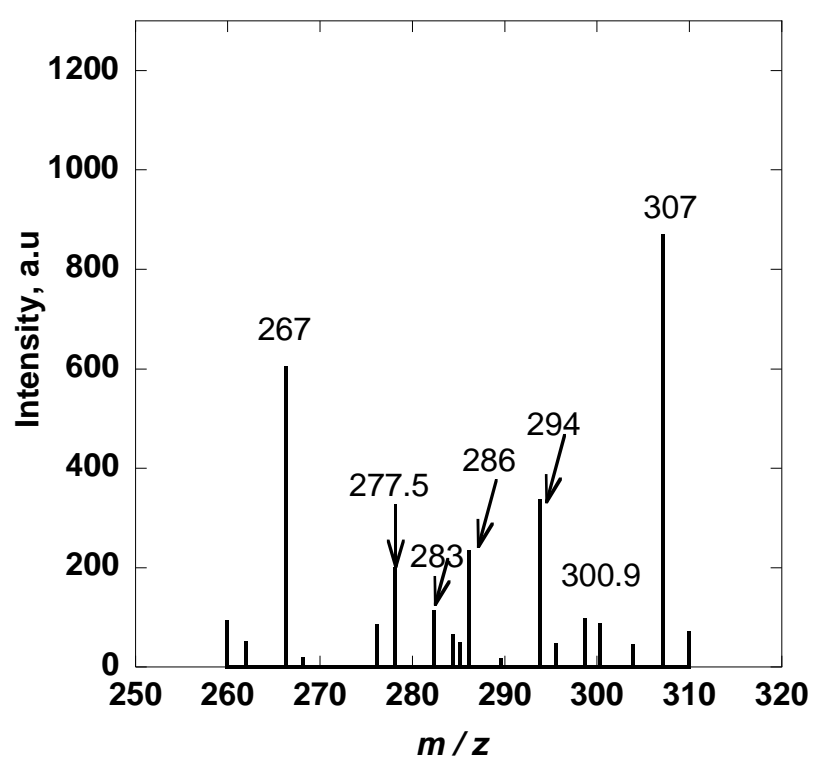

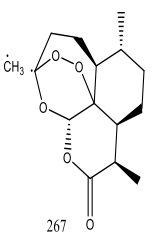

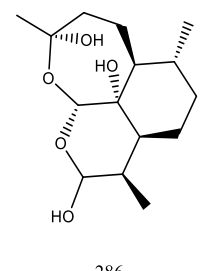

286
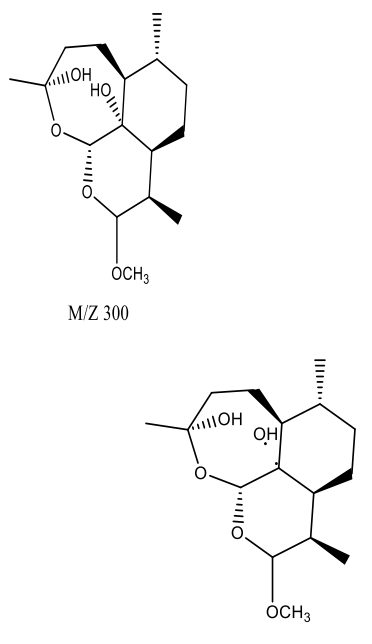

283

Figure 9. Mass spectrum of ART after three hours of electrolysis (peak at 21.5)

Figure 9 shows the mass spectrum of the new chromatographic peak at 21.5 minutes. New fragments after three hours of electrolysis include, $m / z 286,267,307$, and 283 . The new ART derivatives formed after electrochemical reduction all seem to involve either the endo-peroxide bond, or the ketone group on one of the three rings. We think this chromatographic peak is derived from further electrochemical reduction of dihydroartemisinin on the electrode surface. The fact that it is eluted much faster than either ART or dyhidroartemisinin indicate that it is more polar. Hydrolysis of the endo-peroxide bridge makes the resultant molecule more polar, hence eluted much faster using reverse phase chromatography.

However, from our mass spectrometry results it is not clear whether the endo-peroxide bridge or the carboxyl groups were hydrolyzed first during bulk electrolysis. On the other hand, the electrochemistry experiment shows clearly new peaks formed as function of time. The scan rate dependence studies point to a more complex ECE reaction mechanism. Electrochemical and chromatographic methods were used for the first time to determine the products of the electrochemical oxidation. 


\section{Conclusions}

We have studied the electrochemical reduction of ART on a glassy carbon electrode. Initially, reduction of ART on a glassy carbon electrode generated only one reduction peak wave, but on continuous reduction, the reduction product is further reduced to several other electro-generated products. The electro-reduction on glassy carbon electrode was under diffusion control. Chromatographic analysis of bulk reduction products showed continuous growth of new chromatographic peaks. Mass spectrometry analysis showed initial reduction product to be dyhidroartemisinin. Continued reduction generated more products that appeared to target the oxygen bridge to form alcohols. Dihydroartemesinin, although being the major product of electroreduction appeared to transform to other more polar molecule, pointing to hydrolysis of the endoperoxide bridge. Further studies are underway to establish the enzyme derived ART byproducts and compare with those electro-generated.

Acknowledgements: We would like to acknowledge the department of Chemistry and Biochemistry Rowan University for support of this project.

\section{References}

[1] E. Van Geldre, A. Vergauwe, E. Van Den Eeckhout, Plant Molecular Biology 33 (1997) 199-209.

[2] V. Dhingra, C. Rajoli, M.L. Narasu, Bioresource Technology 73 (2000) 279-282.

[3] D. L. Klayman, Science 228 (1985) 1049-1055.

[4] S. R. Meshnick, International Journal for Parasitology 32 (2002) 1655-1660.

[5] S. Singh, A. Giri, S. Giri, Mutation Research/Genetic Toxicology and Environmental Mutagenesis 777 (2015) 1-6.

[6] T. J. C. Anderson, Molecular Biology and Evolution 34 (2017) 131-144.

[7] C. Prosser, Plos One 13 (2018).

[8] L. Dembele, Scientific reports 7 (2017).

[9] A. Hamacher-Brady, H. A. Stein, S. Turschner, I. Toegel, R. Mora, N. Jennewein, T. Efferth, R. Eils, N. R. Brady, Journal of Biological Chemistry 286 (2011) 6587-6601.

[10] N.-D. Yang, S.-H. Tan, S. Ng, Y. Shi, J. Zhou, K. S. W. Tan, W-S. F. Wong, H-M. Shen, Journal of Biological Chemistry 289 (2014) 33425-33441.

[11] J. Suberu, L. Song, S. Slade, N. Sullivan, G. Barker, A. A. Lapkin, Journal of Pharmaceutical and Biomedical Analysis 84 (2013) 269-277.

[12] A. F. T. F. Waffo, Sensors and actuators B: Chemical 275 (2018) 163-173.

[13] J. Zhou, X. Sun, K. Wang, International Journal of Electrochemical Science 11 (2016) 3114-3122.

[14] C. Debnath, Journal of the Korean Chemical Society 55 (2011) 57-62.

[15] J. Ginja Teixeira, Electrochimica Acta 108 (2013) 51-65.

[16] F. Zhang, D.K. Gosser Jr, S. R. Meshnick, Biochemical Pharmacology 43 (1992) 1805-1809.

[17] X. Yang, Tian Gan, X. Zheng, D. Zhu, A. K. Wu, Bulletin of Korean Chemical Society 29 (2008) 1368-1390.

[18] J. Jastrebova, K. E. Markides, L. Nyholm, Y. Bergqvist, Analyst 123 (1998) 313-317.

[19] F-C. Gong, Z-D. Xiao, Z. Cao, D-X. Wu, Talanta 72 (2007) 1453-1457.

[20] R. Jain, Vikas, Colloids and Surfaces B: Biointerfaces 88 (2011) 729-733.

[21] P-H. Yang, Z-J. Zhou, J-Y. Cai, Colloids and Surfaces A: Physicochemical and Engineering Aspects 257258 (2005) 467-472.

[22] J. R. M. Reys, P. R. Lima, A. G. Cioletti, A. S. Ribeiro, F. C. De Abreu, M. O. F. Goulart, L. T. Kubota, Talanta 77 (2008) 909-914.

[23] C. Debnath, P. Saha, A. Ortner, Electroanalysis 20 (2008) 1549-1555.

[24] S. Hosseini, ChemElectroChem (2018) Ahead-of Print.

[25] M. Wilken, S. Ortgies, A. Breder, I. Siewert, ACS Catalysis 8 (2018) 10901-10912.

[26] F. Zheng, Electrochimica Acta 296 (2019) 1095-1101.

[27] E. T. Martin, C. M. Mcguire, M. S. Mubarak, D. G. Peters, Chemical Reviews 116 (2016) 15198-15234.

[28] T. Ericsson, Xenobiotica 44 (2014) 615-626. 
[29] A. M. Mugweru, A. Shore, H. K. Kahi, G. N. Kamau, International Journal of Chemical Kinetics 48 (2016) 72-78.

[30] Z. Mazzochette, Analytical +Methods 9 (2017) 2997-3002.

[31] A. Djimdé, G. Lefèvre, Malaria Journal 8 (2009) S4.

(C2019 by the authors; licensee IAPC, Zagreb, Croatia. This article is an open-access article distributed under the terms and conditions of the Creative Commons Attribution license (http://creativecommons. org/licenses/by/4.0/) 